\title{
Changing trends in seroprevalence rates of transfusion-transmitted diseases among blood donors in Jordan
}

\author{
Lina Souan* ${ }^{*}$, Mahmoud Siag, Hala Al-Salahat, Tareq Al-Atrash and Maher A. Sughayer
}

\begin{abstract}
Background: Hepatitis B and C infections and transmission are a serious challenge to all healthcare systems. We studied seroprevalence rates of Transfusion Transmitted Diseases (TTD) among blood bank donors in Jordan from 2014 to 2019 as a follow-up study of our previously published work. In addition, we wanted to explore the efficacy of the mandatory vaccination of infants against hepatitis B virus (HBV) which was implemented by the Ministry of Health since 1995 for the eradication of HBV infection in Jordan.

Methods: We reviewed blood bank donors' records at King Hussein Cancer Center (KHCC) from January 1st, 2014, until December 31st, 2019. Results of seropositivity prevalence rates for HBsAg, anti-HBcore, and anti-HCV, using Enzyme-Linked ImmunoSorbent Assay (ELISA) were compared to seropositivity rates from our previously published data. In addition, our results were compared to data obtained from other blood banks in Jordan, as well as compared to published information from blood banks in neighboring countries.

Results: The prevalence rates (\%) of seropositive blood donors for viral hepatitis for the years 2014, 2015, 2016, 2017, 2018, and 2019, were as follows: HBsAg rates were 0.3386, 0.2108, 0.1801, 0.1898, 0.2068, and 0.2741; antiHBcore rates were 4.1112, 3.2271, 2.9748, 2.8405, 2.6879 and 3.0986; and anti-HCV rates were $0.1129,0.0486,0.0548$, $0.0654,0.0782$, and 0.0839 , respectively. There was a significant increase in the prevalence of HBsAg, Anti-HBcore and Anti-HCV antibodies in 2019 (one sample z-score test, $p<0.00001$ ).

Conclusions: Prevalence rates of hepatitis B and $C$ infections among Jordanian blood bank donors showed a steady decline between 2009 and 2017, and these rates were much lower in Jordan than in neighboring countries. However, an increase in the prevalence rates of hepatitis B and C infections among blood bank donors was documented in 2019. While the reasons for this increase are not clear yet, these findings highlight the importance of renewed efforts to increase public health awareness of HBV and implement effective measures to prevent the transmission and infection with HBV, including national vaccination programs.
\end{abstract}

Keywords: Donors, Transfusion-transmitted disease, Anti-HBcore antibody, Anti-HCV, Herd immunity, vaccine

\footnotetext{
* Correspondence: Isouan@KHCC.JO; LSOUAN@KHCC.JO

Department of Pathology Laboratory Medicine, King Hussein Cancer Center,

Al-Jubeiha, Amman, Jordan
}

C C The Author(s). 2021 Open Access This article is licensed under a Creative Commons Attribution 4.0 International License, which permits use, sharing, adaptation, distribution and reproduction in any medium or format, as long as you give appropriate credit to the original author(s) and the source, provide a link to the Creative Commons licence, and indicate if changes were made. The images or other third party material in this article are included in the article's Creative Commons licence, unless indicated otherwise in a credit line to the material. If material is not included in the article's Creative Commons licence and your intended use is not permitted by statutory regulation or exceeds the permitted use, you will need to obtain permission directly from the copyright holder. To view a copy of this licence, visit http://creativecommons.org/licenses/by/4.0/ The Creative Commons Public Domain Dedication waiver (http://creativecommons.org/publicdomain/zero/1.0/) applies to the data made available in this article, unless otherwise stated in a credit line to the data. 


\section{Introduction}

Screening of donated blood products for infectious diseases such as hepatitis is crucial to prevent the transmission of diseases. In our previously published study on the seropositivity rates of $\mathrm{HBsAg}$, anti-HBcore, and antiHCV among healthy Blood Bank donors at King Hussein Cancer Center (KHCC), between the years 2009-2013, we found that these rates were lower in these donors relative to published data in neighboring countries $[1,2]$. Moreover, none of these donors in our previous study tested positive for anti-HIV I/II, anti-HTLV I/II, or antiTP [2].

In this study we wanted to make a six-year follow-up on our previously published work in light of the current changes in the country and region demographics. Furthermore, we explored whether the introduction of a mandatory HBV vaccine in 1995 and the gradual entry of vaccinated donors into the donor's pool starting in 2013 may have led to a significant decrease in seropositivity rates of HBV among blood donors from 2014 to 2019. We have undertaken therefore this new study to explore the prevalence rates of seropositive donated blood for HBsAg, anti-HBcore, and anti-HCV, from the years 2014 to 2019, among KHCC blood bank donors.

In addition, we compared our data from this study with data collected from all national blood banks in Jordan; an initiative that hasn't been done or published before. Having updated epidemiological data is important in enrolling and retaining safe blood donors, while knowing the prevalence of infectious markers in the general population aids in recognizing low-risk donor populations [3].

\section{Materials and methods}

The clinical Immunology laboratory at King Hussein Cancer Center (KHCC) performs the screening of blood bank donor samples following the guidelines of the College of American Pathologists' [4]. Enzyme-linked immunosorbent assay (ELISA) technique is used for the majority of the serological screening tests such as the anti-HCV, HBsAg, and anti-HBcore. As for the Jordanian national blood bank and all blood bank centers in Jordan, they use the ELISA technique as well for screening their blood bank donor-samples. Subjects:

A retrospective cross-sectional study was done on 88 , 565 blood and apheresis-platelet healthy donors at KHCC between January 2014 and December 2019. The donors were adults ranging in age from 18 to 60 years of age. All blood and platelet donors were either volunteers or family replacement Jordanian citizens and were interviewed according to standard international blood donor rules and regulations. Blood bank donor-samples were processed as in our previous published work [2].
Data collected from KHCC healthy donors was compared with data collected from blood banks around Jordan. Raw data from the National-Central Blood Bank at Al-Basheer hospital, National Blood Bank (NBB) branches in Amman, NBB in Zarqa, NBB in Irbid, NBB in Al Karak, Islamic Hospital Blood Bank, Jordan University blood bank, University of Science and Technology Blood bank, Jordanian Royal Medical Services blood bank were shared with us by the National-Center Blood bank. Data from all these blood banks was added together and listed under one group titled "National Blood Bank". The total number of blood donors from all national blood banks during the same period was 1,224 , 933 donors.

\section{HBsAg screening}

Screening for HBsAg was performed as in [2]. The specificity of Murex HBsAg Version 3 is estimated to be $99.97 \%(12,326 / 12330)$ with a $95 \%$ confidence interval of $99.92 \%(12,320 / 12330)$ to $99.99 \%(12,328 / 12330)$ by the binomial distribution [5]. The sensitivity of the Bio-Rad Monalisa HBsAg sandwich ELISA test is shown to be $100 \%$ with a specificity on a total of 9894 random blood donors from 3 different sites equals to $99.94 \%$ (9887/ 9893) [6]. The ARCHITECT HBsAg Qualitative II assay is designed to have a specificity of greater than $99.5 \%$ on blood donor specimens [7]. Roche HBsAg Qualitative assay test sensitivity was reported to be $100 \%$ with a specificity of $99.98 \%$ [8].

\section{Anti-HBcore antibody screening}

Screening for Anti-HBcore antibodies was performed using Bio-Rad Monolisa Anti-HBc PLUS assay kit (BioRad, Marnes-la-Coquette, France) as detailed in [2]. The diagnostic sensitivity, performed on 430 positive samples with EIA reference test for the Bio-Rad Monolisa AntiHBc PLUS assay test is $99.53 \%$ (428/430). The specificity of the test on 5071 samples in non-selected blood bank donors is 99.9. The specificity of the test evaluated on 439 patients is 99.5\% [9]. The sensitivity of the ARCHIT ECT Anti-HBc II assay was evaluated with a fourmember panel that was standardized against reference serum from the Paul-Ehrlich-Institute (PEI). The ARCH ITECT Anti- HBC II assay test is designed to show an analytical sensitivity of less than $1.0 \mathrm{PEI}-\mathrm{U} / \mathrm{mL}$. The panel was tested with three reagent lots. The ARCHIT ECT Anti-HBc II assay sensitivity ranged from 0.4 to 0.5 PEI U/mL. The ARCHITECT Anti-HBc II assay is designed to have an overall specificity of $\geq 99.5 \%$ on a blood donor population and $\geq 98.0 \%$ on a hospitalized or diagnostic population [10]. The Roche Anti-HBc antibody assay test sensitivity is reported to be $100 \%$ with a specificity of $99.7 \%$ [11]. 


\section{Anti-HCV antibody screening}

Screening for anti-HCV antibody was performed using DIAsource anti-HCV V 4.0 (DIA-source ImmunoAssays SA, Nivelles, Belgium) ELISA kits as in [2]. Testing procedure and interpretation of the results were done according to the manufacturer's instructions. Samples giving equivocal (gray zone) readings were confirmed by repeating the sample in duplicate using the same kit and testing it using another methodology such as the CMIA on Abbott Architect i1000 analyzer, ARCHITECT Anti-HCV assay kit (ABBOTT, Wiesbaden, Germany), After February 13, 2018, confirmatory tests were performed on Cobas e601 Roche analyzer 6000, Roche Anti-HCV antibody assay kit (Roche Diagnostics International Ltd., Switzerland) using Electrochemiluminescence Method (ECLIA) method. Repeatedly positive samples using the ELISA method were considered positive for Anti-HCV antibody.

The diagnostic sensitivity of the DIAsource ImmunoAssays SA Anti-HCV Elisa V 4.0 test was determined to be $100 \%$ with an analytical specificity of $99.8 \%$ [12]. The overall specificity for the ARCHITECT Anti-HCV assay test was $99.60 \%(10,361 / 10,403)$ with a $95 \%$ confidence interval of 99.45 to $99.71 \%$. The specificity observed ranged between $99.20 \%(496 / 500)$ to $99.70 \%$ (1994/2000). The sensitivity was $99.10 \%$ with a $95 \%$ confidence interval of 96.77 to $99.89 \%$ [13]. As for the Roche Anti-HCV antibody assay the diagnostic sensitivity was $100 \%$. The $95 \%$ lower confidence limit was 99.61\%. The diagnostic specificity of the Elecsys AntiHCV II assay in a group of hospitalized patients was found $99.66 \%$. The $95 \%$ lower confidence limit (2-sided) was $99.41-99.82 \%$ [14].

\section{Statistical methods}

We performed a descriptive data analysis of the results of blood donors' screening tests for HBsAg, Anti-HBV core antibody, and Anti-HCV antibody, and the data are presented as counts and percentages for both $\mathrm{KHCC}$ and national blood banks. Comparison between the current period (2014 to 2019) with the previously reported period (2009 to 2013) was carried out using t-test to determine if there is a significant difference between the means of two groups.

Comparisons between years (2009 to 2018) in the KHCC donor screening tests (HBsAg, Anti-HBcore, and Anti-HCV) was carried out using Chi square test or Fisher exact depending on the assumption required for each test. The same was done to compare positive results of HBsAg and Anti-HCV in the KHCC and $\mathrm{Na}-$ tional $\mathrm{BB}$ donor populations according to year.

Trend was assisted and presented using Joinpoint regression program, and Annual Percent Change (APC) was reported in addition to significant critical points.

A significance criterion of $p<=0.05$ was used in the analysis. All analyses were performed using SAS version 9.4 (SAS Institute Inc., Cary, NC).

One-sample z-test was used to determine whether the population mean of the prevalence rates of seropositivity from 2016 to 2019 is significantly different from the mean of the 2019 sample population. Hence, it is unlikely to have occurred by chance.

\section{Results}

The total number of healthy Jordanians who donated blood or platelets at KHCC blood bank from the years

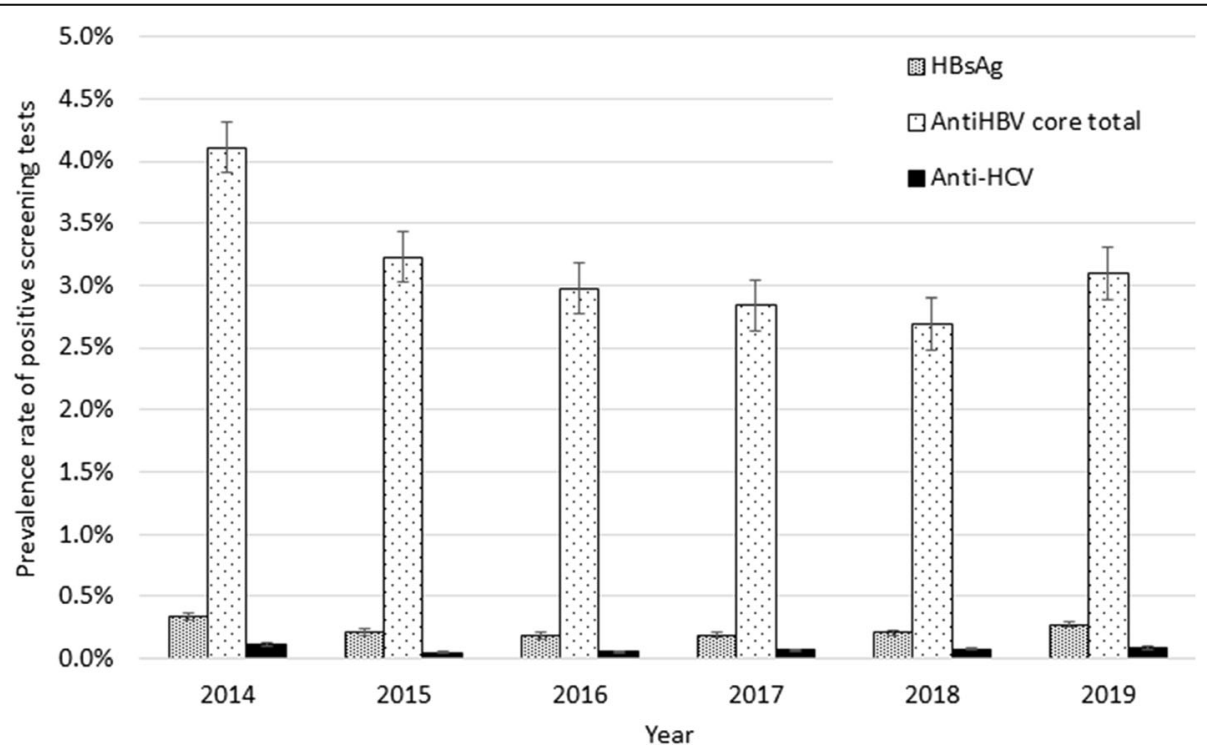

Fig. 1 Percent Prevalence of HBsAg; Anti-HBV core total; Anti-HCV among KHCC blood Bank Donors (error bars represent standard error) 
Table $1 \mathrm{KHCC}$ and national blood bank donors-prevalence rates and $p$ value for $\mathrm{HBsAg}$ and anti-HCV TTD markers from the year 2014-2019

\begin{tabular}{lccccccc}
\hline TDD marker & $\mathbf{2 0 1 4}$ & $\mathbf{2 0 1 5}$ & $\mathbf{2 0 1 6}$ & $\mathbf{2 0 1 7}$ & $\mathbf{2 0 1 8}$ & $\mathbf{2 0 1 9}$ & $\boldsymbol{p}$-value \\
\hline HBsAg & & & & & & & \\
National BB & $0.46 \%$ & $0.41 \%$ & $0.40 \%$ & $0.34 \%$ & $0.29 \%$ & $0.30 \%$ & 0.99 \\
KHCC & $0.30 \%$ & $0.21 \%$ & $0.18 \%$ & $0.19 \%$ & $0.21 \%$ & $0.27 \%$ & \\
HCV & & & & & & & \\
National BB & $0.11 \%$ & $0.13 \%$ & $0.12 \%$ & $0.10 \%$ & $0.10 \%$ & $0.09 \%$ & 0.99 \\
KHCC & $0.11 \%$ & $0.05 \%$ & $0.05 \%$ & $0.07 \%$ & $0.08 \%$ & $0.08 \%$ & \\
\hline
\end{tabular}

2014 to 2019 was 88,565 donors while the number of donors at all other national blood banks was 1,224,933 donors during the same study period. All individuals were adults whose age ranged from 18 to 60 years and were randomly distributed among both genders.

Figure 1 demonstrates the prevalence rates of $\mathrm{HBsAg}$ seropositivity among KHCC donors from 2014 to 2019. The prevalence rate was 0.3386, 0.2108, 0.1801, 0.1898, $0.2068,0.2741 \%$ respectively, while the prevalence rates of anti-HBV core total antibodies were 4.1112, 3.2271, $2.9748,2.8405,2.6879,3.0986 \%$ respectively. The prevalence rates of anti-HCV during the study period at KHCC was $0.1129,0.0486,0.0548,0.0654,0.0782$, and $0.0839 \%$.

The prevalence rates of the TTD among blood donors among all blood banks in Jordan were as follows: for HBsAg it was 0.46, 0.4143, 0.4013, 0.3385, 0.2929, $0.3039 \%$ between the years 2014 and 2019 respectively. While the prevalence rates of anti-HCV during the study period was $0.11,0.1334,0.1309,0.1033,0.1004,0.0936 \%$, respectively. As for prevalence rates of anti-HBV core total antibodies, they were available for 2019 only and it was $3.2045 \%$. Table 1 shows the comparison between KHCC and national blood bank donors' prevalence rates for HBsAg and anti-HCV from the years 2014 to 2019 with the associated $p$-values.

Table 2 illustrates the numbers of positive blood bank donors for the HBsAg and anti HCV-TTD markers among KHCC (A) from the year 2009 to 2019 and national blood bank donors (B) from the year 2015 to 2019 because no raw data was available for the national blood bank donors. The prevalence rates were provided for the earlier years.

We used the Joinpoint regression analysis describing the annual percent change (APC) in comparing our data from our previous publication with our current data. We found that the prevalence rate of HBsAg continued to show a significant decrease with an APC value of - 16.27 between the years 2009 to 2017 (Fig. 2). The APC was 16.3 with a lower Confidence Level (CL) equals to -20.6 and an upper CL equals to -11.7 and a $p$-value of 0.0 between the years 2009-2017. While the APC was 24.8 with a lower CL of -23 and an Upper CL equals to 102.4 and a $p$-value of 0.3 between the years 2017 to 2019 (Fig. 2).

Furthermore, there was no significant difference seen in the calculated prevalence rate among national blood bank donors compared to KHCC blood bank donors for HBsAg marker (Fig. 3). The APC was - 9.1 with a lower CL equal to -12.2 and an upper CL equal to -5.9 and a $p$-value of 0.0 between the years 2014 to 2019 for the national blood bank donors. For KHCC blood bank donors, the APC was -1.5 with a lower CL of -15.1 and an upper CL equal to 14.3 and a $p$-value of 0.8 between the years 2014 to 2019 (Fig. 3).

Interestingly, there was a similar significant decrease measured in the prevalence rate of HBsAg among blood donors in Jordanian national blood banks similar to what was observed at KHCC from the years 2011 to 2019 with an APC value of -9.51 (Fig. 4). The APC was - 9.5 with

Table 2 KHCC and national blood bank donors-prevalence rates and $p$ value for HBsAg and anti-HCV TTD markers from the year 2014-2019

\begin{tabular}{|c|c|c|c|c|c|c|c|c|c|c|c|}
\hline \multicolumn{12}{|l|}{ A:KHCC } \\
\hline \multirow[t]{2}{*}{ TDD marker } & \multicolumn{11}{|l|}{ Year } \\
\hline & 2009 & 2010 & 2011 & 2012 & 2013 & 2014 & 2015 & 2016 & 2017 & 2018 & 2019 \\
\hline $\mathrm{HBsAg}$ & 62 & 79 & 78 & 63 & 51 & 42 & 26 & 23 & 29 & 37 & 49 \\
\hline AntiHBV core total & 731 & 922 & 846 & 762 & 518 & 510 & 398 & 380 & 434 & 481 & 554 \\
\hline AntiHCV & 20 & 26 & 18 & 28 & 14 & 14 & 6 & 7 & 10 & 14 & 15 \\
\hline total & 10,101 & 12,694 & 13,387 & 14,256 & 12,495 & 12,405 & 12,333 & 12,774 & 15,279 & 17,895 & 17,879 \\
\hline \multicolumn{12}{|c|}{ B: National Blood Bank } \\
\hline \multirow[t]{2}{*}{ TTD marker } & \multicolumn{11}{|l|}{ Year } \\
\hline & 2015 & 2016 & 2017 & 2018 & 2019 & & & & & & \\
\hline $\mathrm{HBsAg}$ & 840 & 782 & 721 & 672 & 694 & & & & & & \\
\hline AntiHCV & 269 & 166 & 200 & 235 & 211 & & & & & & \\
\hline total & 208,798 & 201,426 & 220,584 & 237,112 & 230,846 & & & & & & \\
\hline
\end{tabular}




\section{$1: 1$ Joinpoint}

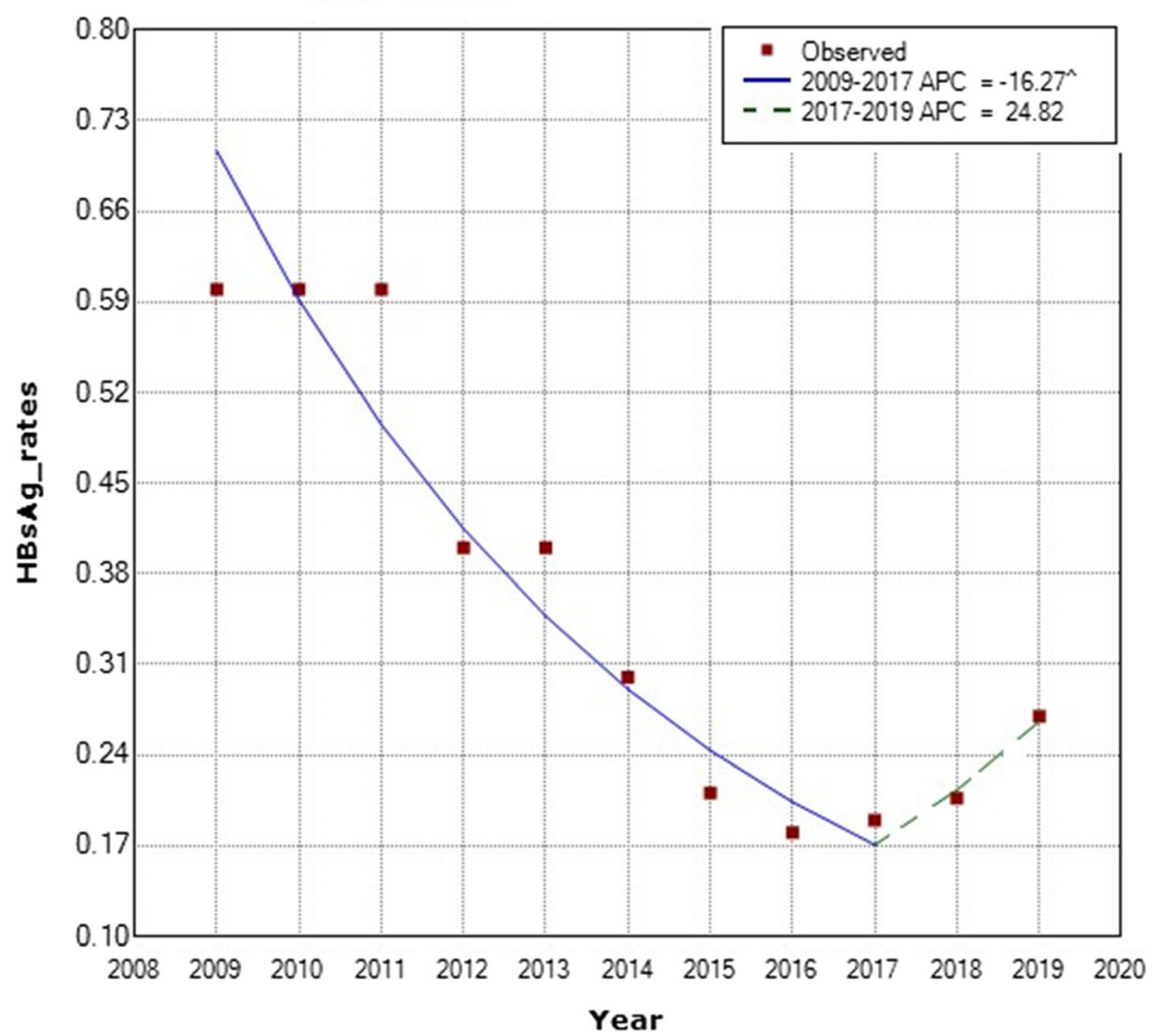

Fig. 2 Annual Percent Change (APC) in the prevalence of HBsAg infection among healthy KHCC-blood bank donors from 2009 to 2019. (The Annual Percent Change (APC) is significantly different from zero at alpha $=0.05$ )

a lower $\mathrm{CL}$ equal to -10.9 and an upper $\mathrm{CL}$ equal to 8.1 with a $p$-value of 0.0 between the years 2011-2019 for the national blood bank donors (Fig. 4).

On the other hand, there was a slight, yet significant increase in the prevalence of HBsAg infection among KHCC-donors in 2019 using single sample Z score ( $p$ value is $<0.00001$ ) observed in both KHCC and national blood bank donors (Figs. 2 and 4).

As for the anti-HBcore antibodies, our data showed that there was a continuous significant decrease in the prevalence of anti-HBV core total antibodies among healthy-KHCC blood bank donors from the year 2009 to 2017 (APC $=-12.69)$ (Fig. 5). However, there was a slight yet significant increase in the prevalence of antiHBcore antibodies among KHCC-donors in 2019 using single sample $\mathrm{Z}$ score ( $p$ value is $<0.00001)$. No comparison was made with the Jordanian national blood bank-donors because this test was implemented in those centers in 2019; hence, there was insufficient data available for comparison.

The APC was -12.7 with a lower CL equals to -15 and an upper CL equal to -10.3 and a $p$-value of 0.0 between the years 2009 to 2017. While the APC was 7.6 with a lower CL of -16 and an upper CL equal to 37.7 and a $p$-value of 0.5 between the years 2017 to 2019 (Fig. 5).

Furthermore, our results showed a continuous significant decrease in the prevalence of anti-HCV antibodies among healthy-KHCC blood bank donors from the years 2009 to 2016 (APC $=-17.89)$ (Fig. 6). The APC was 17.9 with a lower $\mathrm{CL}$ equal to -28.8 and an upper $\mathrm{CL}$ equal to -5.3 with the $p$-value of 0.0 between the years 2009 and 2016. For the years 2016 to 2019, the APC was 15 with a lower $\mathrm{CL}$ of -32.5 and an upper $\mathrm{CL}$ equal to 96.1 and a $p$-value of 0.5 (Fig. 6).

On the other hand, there was a minor-significant increase in the prevalence of anti-HCV antibodies among KHCC-donors in 2019 using single sample Z score ( $p$ value is <0.00001) (Fig. 6).

The same pattern was observed among Jordaniandonors from national blood banks around Jordan where there was a significant decrease in the prevalence of anti-HCV antibodies observed among healthy donors from the year 2011 to 2019 (APC = -3.27) (Fig. 7). The 
2: 0 Joinpoints versus $1: 0$ Joinpoints

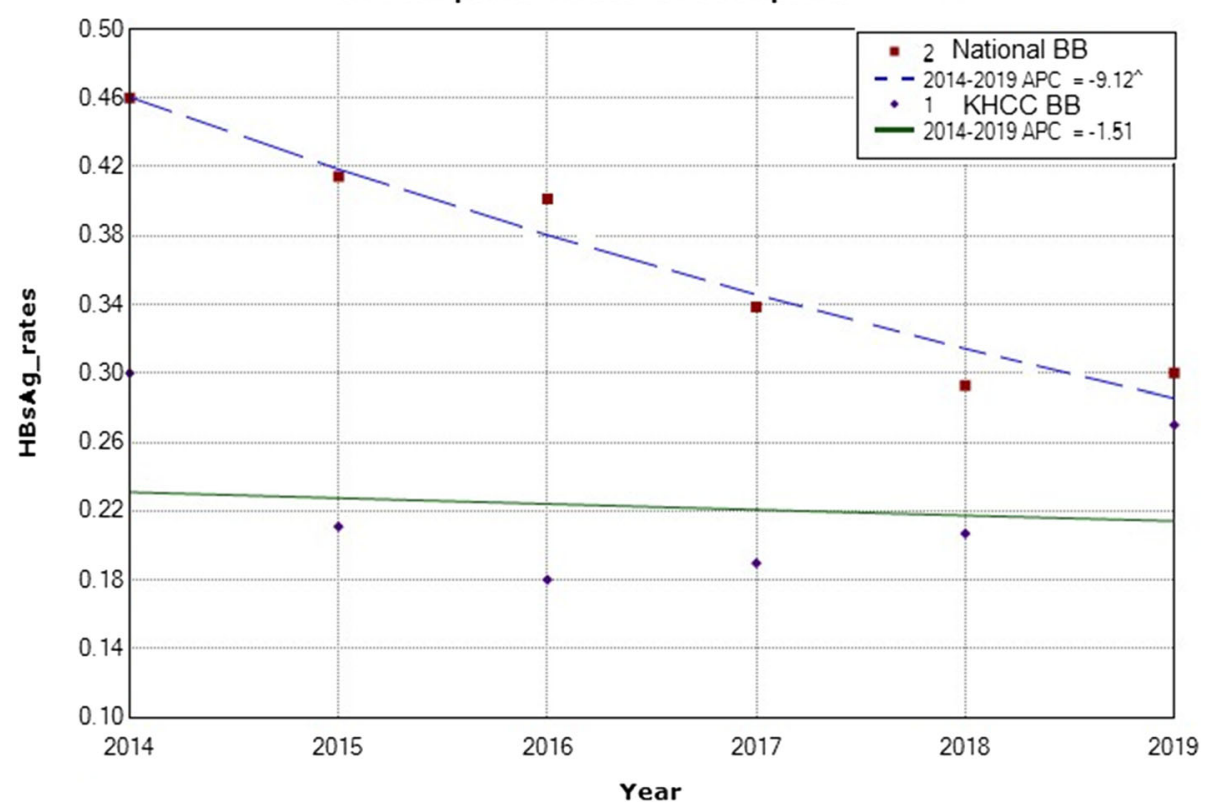

Fig. 3 Annual Percent Change (APC) in the prevalence of HBsAg infection among healthy national blood bank donors compared to KHCC blood bank 2014 to 2019. (The Annual Percent Change (APC) is significantly different from zero at alpha $=0.05$ )

APC was -3.3 with a lower CL equals to -6.8 and an upper CL equals to 0.4 and a $p$-value of 0.1 between the years 2011 to 2019 (Fig. 7).

\section{Discussion}

Our data demonstrate for the first time a significant steady decrease in the prevalence rates of HBsAg positivity among all Jordanian blood bank donors between the years 2009 to 2017. Furthermore, we show that there was no significant differences in the prevalence rates of $\mathrm{HBsAg}$ and anti-HCV seropositivity in donated blood as a proxy for active hepatitis $\mathrm{B}$ and $\mathrm{C}$ infections, respectively, among donors at KHCC blood bank compared with other donors at national blood bank branches in Jordan.

In addition, our results still support our previous finding that seroprevalence rate of HBsAg in Jordan is the lowest compared to neighboring countries. For example, a study performed in Duhok, Iraq, where the researchers show that the prevalence rate was $1.14 \%$ for $\mathrm{HBsAg}$ positive (HBsAg and anti-HBcore positive) and $8.2 \%$ for HBcore positive in 2018 compared to 0.2068 and $2.6879 \%$ respectively in Jordan [15]. Similarly in the Kingdom of Saudi Arabia (KSA), the estimation for HBsAg prevalence in 2019 was around 1.3\% compared to $0.2741 \%$ for the same year in Jordan [16]. In Lebanon on the other hand; the prevalence rate for HBsAg among adults was $1.6 \%$ in a study published in 2007 [17]. The prevalence rates in Egypt were 1.22\% for HBsAg in 2014
[18]. Recent studies showed that the HBsAg pooled prevalence in blood donors of both EMRO and Middle Eastern countries was 2.03\% Furthermore, the prevalence rate in the EMRO countries was $1.99 \%$ while the prevalence rate was $1.62 \%$ in Middle Eastern countries. The prevalence among blood donors with more than one study was $0.24 \%$ in Iran, $2.35 \%$ in Pakistan, and $2.1 \%$ in Yemen [1].

Data concerning the prevalence of seropositive donated blood for anti-HBV core antibodies is scant, because this test is not a mandatory screening test at the majority of blood banks around the world. Nevertheless, a recent study from Saudi Arabia showed that 9.81\% of blood donors at King Khalid General Hospital in Majmaah, Saudi Arabia, were positive for anti-HBc in 2018 [19] compared to $3.0986 \%$ in Jordan.

Moreover, a recent study by Bahat et al. on the prevalence of sexually transmitted diseases among Syrian immigrant pregnant women showed that the rates of $\mathrm{HBsAg}$, anti-HCV, and anti-HIV seropositivity were 1.1, 0.1 , and $0.03 \%$, respectively, between the years 2012 and 2018. Additionally, Turkish women had seropositivity rates of $1.8,0.2$, and $0.08 \%$ for $\mathrm{HBsAg}$, anti-HCV, and anti-HIV, respectively [20]..

The significant decrease in the prevalence rates of HBsAg and anti-HBcore antibodies among blood bank donors in Jordan may be related to the mandatory infant vaccination plan which was started in 1995, and perhaps to other factors such as increased awareness and 


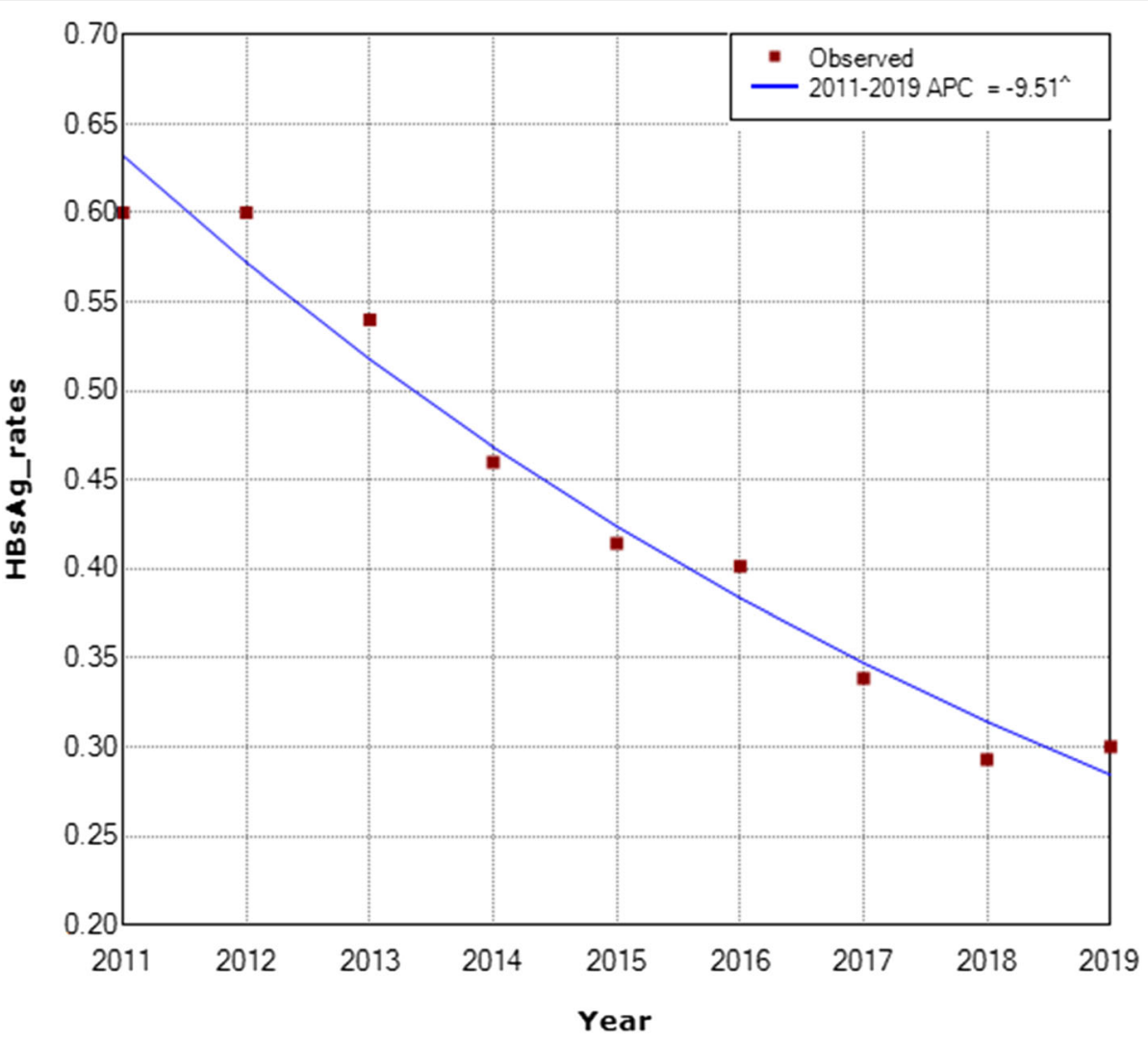

Fig. 4 Annual Percent Change (APC) in the prevalence of HBsAg infection among healthy national blood bank donors between the years 2011 to 2019. (The Annual Percent Change (APC) is significantly different from zero at alpha $=0.05$ )

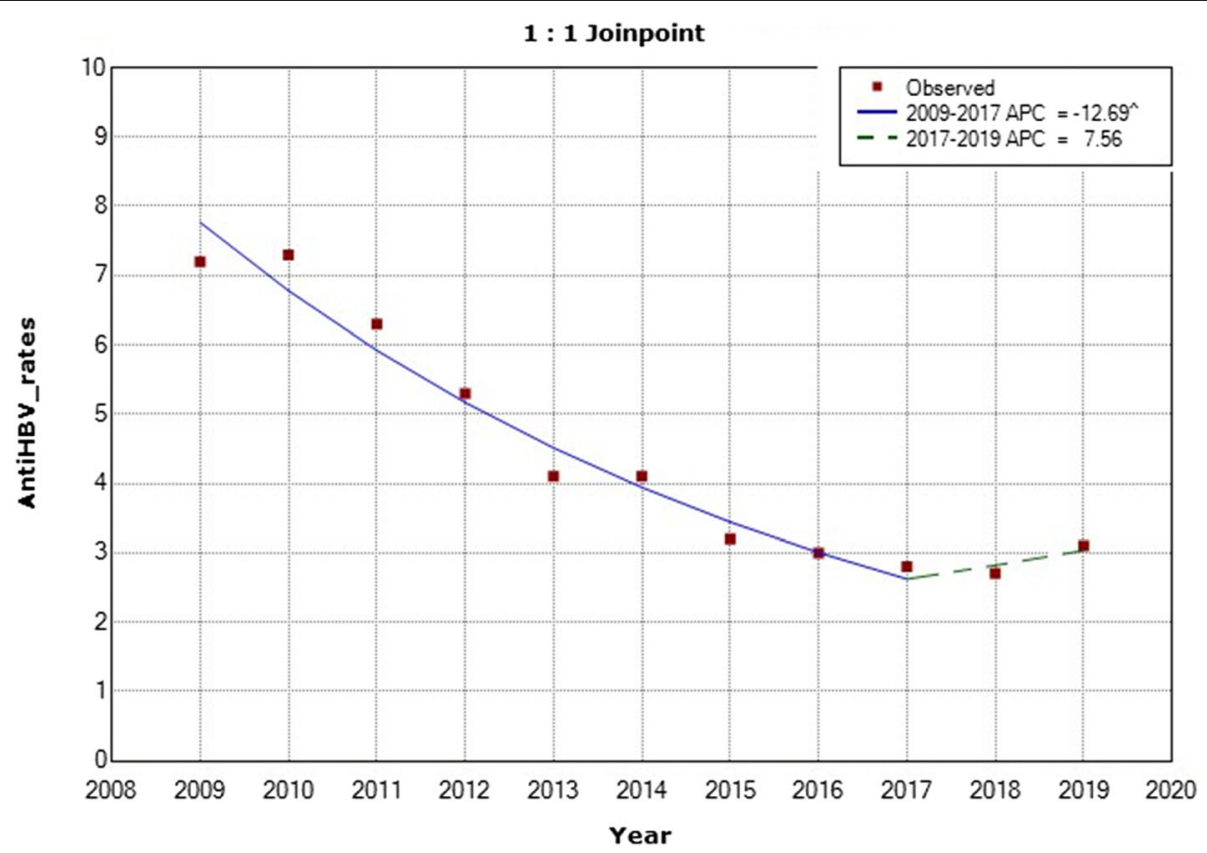

Fig. 5 Annual Percent Change (APC) in the prevalence of anti-HBV core total antibodies among healthy KHCC-blood bank donors from 2009 to 2019. (The Annual Percent Change (APC) is significantly different from zero at alpha $=0.05$ ) 


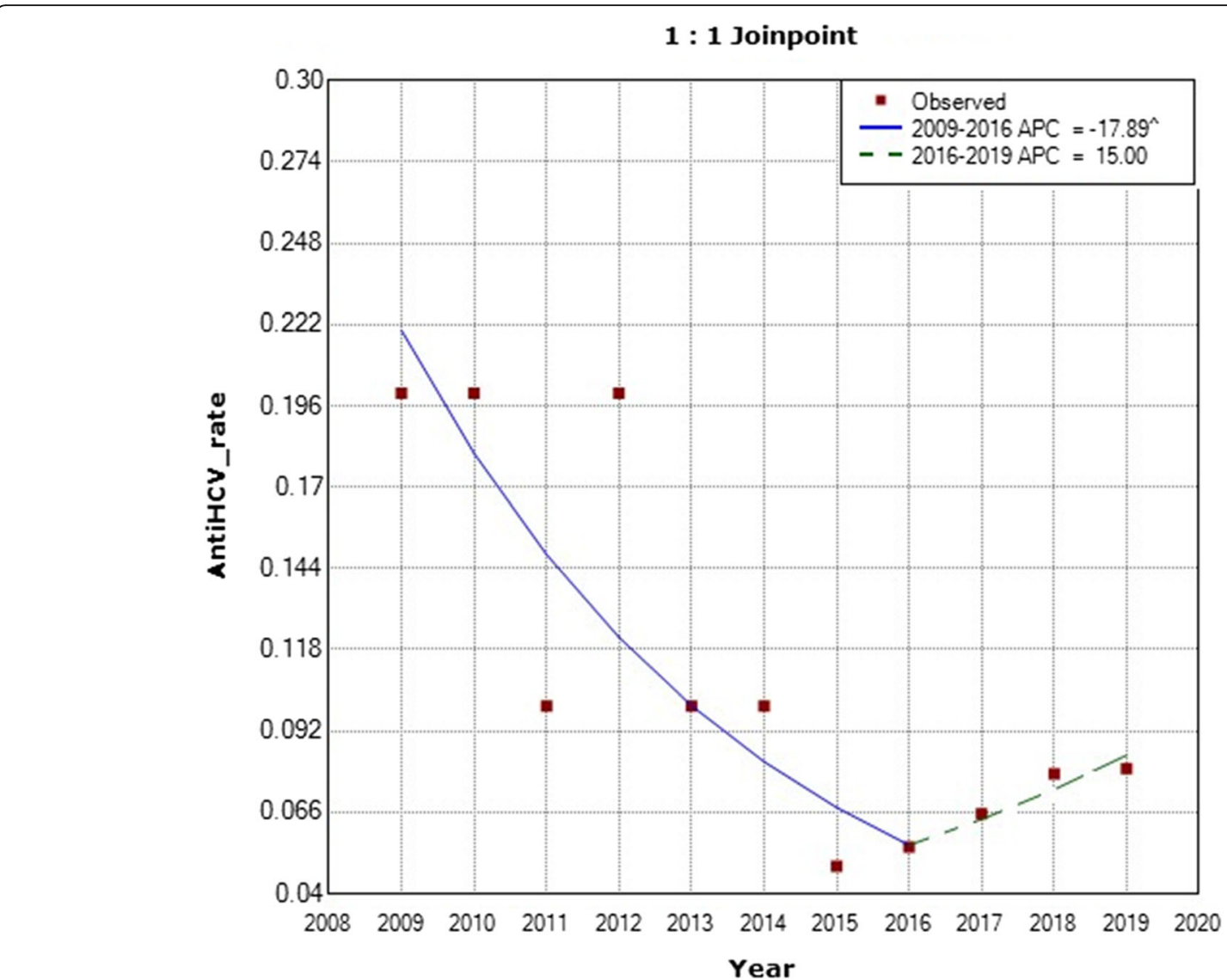

Fig. 6 Annual Percent Change (APC) in the prevalence of anti-HCV antibodies among healthy KHCC-blood bank donors from 2009 to 2019 . (The Annual Percent Change (APC) is significantly different from zero at alpha $=0.05$ )

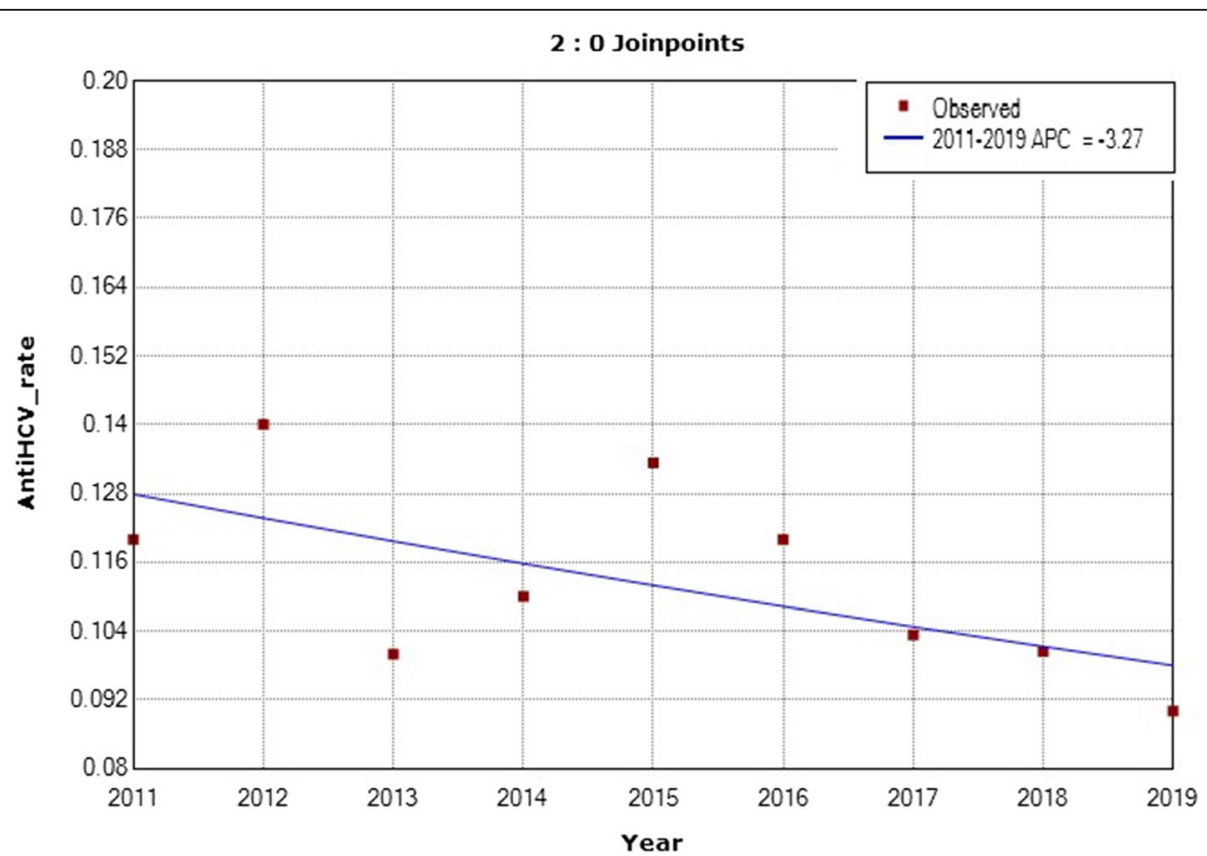

Fig. 7 Annual Percent Change (APC) in the prevalence of anti-HCV antibodies among healthy National-blood bank donors from 2011 to 2019. (The Annual Percent Change (APC) is significantly different from zero at alpha $=0.05$ ) 
practices of proper hygiene $[1,21]$. The effective protective role of anti-HBV vaccination has been documented in several recent publications. Vittal and Ghany demonstrated that vaccination regimens that followed the WHO guidelines have proven to be 90 to $95 \%$ effective in preventing HBV infection and transmission [21]. Data from Qatar has also shown a marked decline in HBV infection rates after vaccination strategies [22].

Prevalence rates of seropositive donated blood at KHCC Blood Bank for anti-HCV in Jordan showed significant steady decline in the years 2009 to 2016 similar to the decline in prevalence rates for $\mathrm{HBsAg}$ and antiHBcore antibody seropositivity $(\mathrm{APC}=-17.89)$ over the same period. Similar observations were made in seropositivty rates for these viral markers among national blood bank donors $(\mathrm{APC}=-3.27)$. Furthermore, rates of $\mathrm{HCV}$ infection showed a significant decline as well. These findings may be explained, at least in part, by improved socioeconomic status and health awareness among Jordanians [23].

With respect to anti-HCV seropositivity in donated blood, we found that Jordan has relatively much lower prevalence rates to the rates in neighboring countries. Rates of seropositivity for $\mathrm{HCV}$ vary between countries and different regions in the same country. The Aseer region in southwestern Saudi Arabia had an anti-HCV prevalence rate of $2.2 \%$ [24], while Alexandria, Egypt, had a high prevalence rate of $23.2 \%$ [25]. In contrast, the prevalence of anti-HCV rate decreased in voluntary donors in Cairo from 4.2 to $1.5 \%$ [18]. In comparison, prevalence rates for anti-HCV were $2.61 \%$ in Pakistan [26], 2.32\% in Mali [27], 3.6\% in Calabar, Nigeria [28], and $0.46 \%$ in Brazil [29].

Interestingly, our data showed a small, yet a statistically significant, increase in the prevalence of $\mathrm{HBsAg}$, Anti-HBcore and Anti-HCV antibodies among blood bank donors in Jordan in the year 2019 compared to the years 2016 to 2018 using the one sample z-test for mean analysis with a $p<0.00001$. The reason for this increase in prevalence rates of hepatitis $B$ and $C$ virus seropositivity in 2019 is very interesting and needs a comprehensive and thorough investigation.

While our study showed for the first time the effectiveness of Jordan's national mandatory HBV vaccination campaign in reducing HBV infection rates among Jordanian citizens until 2018, the recent increase in HBV infection rates among Jordanian citizens suggests a mandatory fast revision of the current national vaccination program implemented by the Ministry of Health. For example, we recommend the implementation of HBV screening programs for Jordanian youths and a serious and rapid revision of the public healthcare measures as well as revision of the national vaccination program including consideration for an HBV booster immunization for Jordanian adolescents to boost their immunity against HBV infection.

Several studies demonstrate the efficacy of infant Hepatitis B vaccination, yet they emphasize the need for a booster shot given to adolescents due to insufficient levels of protective HBV antibodies among these adolescents [30-33]. Furthermore, during the World Hepatitis Summit 2017 and on "World Hepatitis Day 2019" the WHO focused on the theme "Invest in eliminating hepatitis" to highlight the need for increased domestic and international funding to scale up hepatitis prevention, testing, and treatment services, in order to achieve a complete elimination of HBV infections in 2030 [34]. Based on our study, we recommend a greater public health response to HBV infections, such as screening adolescents and young adults for immunity to HBV, followed by administering a booster shot to individuals who are lacking such immunity.

\section{Abbreviations}

HBV: Hepatitis B virus; HCV: Hepatitis C virus; TTD: Transfusion Transmitted

Diseases; HBsAg: Hepatitis B surface antigen; anti- HBc: Hepatitis B core total antibodies; ELISA: Enzyme-Linked ImmunoSorbent Assay; KHCC: King Hussein Cancer Center; APC: Annual Percent Change

\section{Acknowledgments}

The authors wish to thank Ms. Dalia Al-Rimawi and Ms. Ayat Taqash from the Biostatistics Unit, Research Office team at KHCC for performing the statistical analysis of the data. Thanks to the National blood bank head Dr. Asia Al Odwan and Mr. Azzam Abo Al Ruz for their help in sharing the national blood bank data.

In addition, sincere thanks to Drs. Imad and Jane Jarjour for English editing of this manuscript.

\section{Authors' contributions}

Conceptualization: L.S., and M.A.S.; Methodology, Validation, Data Curation: L.S., M.S., T.A. H.A.S.; Formal Analysis: L.S. and M.A.S.; Writing—Original Draft Preparation: L.S.; Writing—Review and Editing: L.S. and M.A.S.; Resources: M.A.S. All authors have read and agreed to the published version of the manuscript.

\section{Funding}

We declare that we did not receive funding nor financial support/grants from any institution, private or corporate entity.

\section{Availability of data and materials}

The datasets used and/or analyzed during the current study are available from the corresponding author on reasonable request.

\section{Declarations}

\section{Ethics approval and consent to participate}

The study was approved by King Hussein Cancer Center-Institutional Review Board, IRB\# 19 KHCC 07, in accordance with the Declaration of Helsinki. KHCC-IRB committee considered the use of data for the research in this study without the need of consent of the study participants since the study was a retrospective study/ chart review and all blood bank donors are consented at the time of donation using the internationally approved blood bank donation consent form.

\section{Consent for publication}

This manuscript does not contain data from any individual person. The KHCC-IRB committee considered the use of data for the research in this study without the need of consent of the study participants since the study was a retrospective study/ chart review and all blood bank donors are consented at the time of donation using the internationally approved blood 
bank donation consent form (IRB\# 19 KHCC 07). Hence, consent for publication is not applicable.

\section{Competing interests}

Authors declare no competing interests.

\section{Received: 30 June 2020 Accepted: 17 May 2021}

Published online: 31 May 2021

\section{References}

1. Babanejad M, Izadi N, Najafi F, Alavian SM. The HBsAg prevalence among blood donors from eastern Mediterranean and middle eastern countries: a systematic review and meta-analysis. Hepat Mon. 2016;16(3):e35664. https:// doi.org/10.5812/hepatmon.35664.

2. Souan L, Tout F, Siag M, Sughayer MA. Seroprevalence rates of transfusiontransmitted infections among blood donors in Jordan. J Infect Dev Ctries. 2016;10(4):377-83. https://doi.org/10.3855/jidc.8160.

3. Chandra T, Rizvi SN, Agarwal D. Decreasing prevalence of transfusion transmitted infection in Indian scenario. ScientificWorldJournal. 2014;2014: 173939.

4. Transfusion Medicine Checklist [https://elss.cap.org/elss/faces/org/cap/elss/ ui/page/elssmain.jspx?_afrLoop $=765207228138376 \&$ afrWindowMode $=0 \&$ _a frWindowld=null\#!\%40\%40\%3F_afrWindowld\%3Dnull\%26_afrLoop\%3D7652 07228138376\%26_afrWindowMode\%3D0\%26_adf.ctrl-state\%3D107ozyq2 9i_4].

5. Murex HBsAg, Version 3 ELISA Infectious disease [package insert]. DiaSorin: Saluggia (VC), Italy; 2005. https://www.diasorin.com/sites/default/files/allega ti_prodotti/Murex\%20HBsAG\%20Version\%203.pdf.

6. Monolisa ${ }^{\text {TM }}$ HBs Ag ULTRA [package insert]. Marnes-la-Coquette: Bio-Rad; 2011. https://www.biorad.com/webroot/web/pdf/inserts/CDG/en/883614_ EN.pdf.

7. HBsAg Qualitative [package insert]. Abbott Park: Abbott; 2011.

8. HBsAg II [package insert]. Indianapolis: Roche Diagnostics; 2017.

9. Monolisa ${ }^{\text {TM }}$ Anti-HBc PLUS [package insert]. Marnes-la-Coquette: Bio-Rad; 2009. https://www.bioRad.com/webroot/web/pdf/inserts/CDG/en/Literature/ inserts/883565_GB.pdf.

10. Anti-HBC II [package insert]. Abbott Park: Abbott; 2008.

11. Anti-HBc, Antibodies to hepatitis B core antigen (anti-HBC) [package insert]. Indianapolis: Roche Diagnostics; 2017

12. Anti-HCV Elisa 4.0 [package insert]. Louvain-la-Neuve: DIAsource ImmunoAssays SA; 2019.

13. Anti-HCV [package insert] Abbott: Abbott Park; 2006

14. Anti-HCV II, Antibody to hepatitis C virus (anti-HCV) [package insert] Indianapolis: Roche Diagnostics; 2017.

15. N RH. Risk factors of hepatitis $B$ virus infection among blood donors in Duhok city, Kurdistan region, Iraq. Caspian J Intern Med. 2018;9(1):22-6. https://doi.org/10.22088/cjim.9.1.22.

16. Aljumah AA, Babatin M, Hashim A, Abaalkhail F, Bassil N, Safwat M, et al. Hepatitis B care pathway in Saudi Arabia: current situation, gaps and actions. Saudi J Gastroenterol. 2019;25(2):73-80. https://doi.org/10.4103/sjg SJG_421_18.

17. Saab BR, Nassar NT, Musharrafieh U, Araj GF, Khogali M. Prevalence of hepatitis B in a presumably healthy Lebanese population. J Med Liban. 2007;55(1):11-4.

18. Hussein E. Blood donor recruitment strategies and their impact on blood safety in Egypt. Transfus Apher Sci. 2014;50(1):63-7. https://doi.org/10.1016/j. transci.2013.11.005.

19. Alaidarous M, Choudhary RK, Waly MI, Mir S, Bin Dukhyil A, Banawas SS, et al. The prevalence of transfusion-transmitted infections and nucleic acid testing among blood donors in Majmaah, Saudi Arabia. J Infect Public Health. 2018;11(5):702-6. https://doi.org/10.1016/j.jiph.2018.04.008.

20. Yalcin Bahat P, Turan G, Yuksel Ozgor B, Bagci Cakmak K. Comparison of hepatitis B, hepatitis $C$, and HIV seropositivity of Syrian and Turkish pregnant women. Turk J Obstet Gynecol. 2019;16(2):95-9. https://doi.org/10.4274/tjod. galenos.2019.15564.

21. Vittal A, Ghany MG. WHO guidelines for prevention, care and treatment of individuals infected with HBV: a US perspective. Clin Liver Dis. 2019;23(3): 417-32. https://doi.org/10.1016/j.cld.2019.04.008.

22. Al Romaihi HE, Ganesan N, Farag EA, Smatti MK, Nasrallah GK, Himatt SM, Derbala MF, Alshamali M, Mahadoon LK, Khogali HS, Sallam M, Al Thani AA, Al Thani M, Al Kaabi S, Yassine HM. Demographics and Epidemiology of
Hepatitis B in the State of Qatar: A Five-Year Surveillance-Based Incidence Study. Pathogens. 2019;8(2):68. https://doi.org/10.3390/pathogens8020068.

23. Daly MC, Duncan GJ, McDonough P, Williams DR. Optimal indicators of socioeconomic status for health research. Am J Public Health. 2002;92(7): 1151-7. https://doi.org/10.2105/ajph.92.7.1151.

24. Al Humayed SM, El-Mekki AA, Mahfouz AA. Hepatitis C virus infection in southwestern Saudi Arabia: are we still in the plateau phase? J Med Virol. 2017:89(5):867-71. https://doi.org/10.1002/jmv.24712

25. Hashish MH, El-Barrawy MA, Mahmoud OA, Abdel Rahman NW. TT virus among blood donors in Alexandria. J Egypt Public Health Assoc. 2005;80(56):651-64.

26. Irfan SM, Uddin J, Zaheer HA, Sultan S, Baig A. Trends in transfusion transmitted infections among replacement blood donors in Karachi, Pakistan. Turk J Haematol. 2013;30(2):163-7. https://doi.org/10.4274/Tjh.2012.0132.

27. Jary $A$, Dienta $S$, Leducq $V$, Le Hingrat $Q$, Cisse $M$, Diarra $A B$, et al. Seroprevalence and risk factors for HIV, HCV, HBV and syphilis among blood donors in Mali. BMC Infect Dis. 2019;19(1):1064. https://doi.org/10.1186/s12 879-019-4699-3.

28. Okoroiwu HU, Okafor IM, Asemota EA, Okpokam DC. Seroprevalence of transfusion-transmissible infections (HBV, HCV, syphilis and HIV) among prospective blood donors in a tertiary health care facility in Calabar, Nigeria; an eleven years evaluation. BMC Public Health. 2018;18(1):645. https://doi. org/10.1186/s12889-018-5555-x.

29. Pessoni LL, Aquino EC, Alcantara KC. Prevalence and trends in transfusiontransmissible infections among blood donors in Brazil from 2010 to 2016. Hematol Transfus Cell Ther. 2019;41(4):310-5. https://doi.org/10.1016/j.htct.2 019.03.009.

30. Arefkhah N, Vafazadeh S, Shahriarirad S, Ghorbani F, Zoghi S, Emami M, et al. Serum levels of anti-hepatitis B surface antibodies among vaccinated children aged 1 to 12 years in a rural community in Fars Province, southern Iran. J Immunoassay Immunochem. 2020;41(1):20-7. https://doi.org/10.1 080/15321819.2019.1675696

31. Di Lello FA, Blejer J, Alter A, Bartoli S, Vargas F, Ruiz R, et al. Hepatitis B surface antibodies seroprevalence among people born before and after implementation of universal HBV vaccination. Vaccine. 2020;38(12):2678-82. https://doi.org/10.1016/j.vaccine.2020.02.014

32. Hefele L, Vannachone S, Khounvisith V, Nouanthong P, Sayasone S, Kounnavong $S$, et al. Lasting benefit of infant hepatitis $B$ vaccination in adolescents in the Lao People's Democratic Republic. Int J Infect Dis. 2020; 93:217-23. https://doi.org/10.1016/j.jijid.2020.01.055

33. Yang $Y T$, Huang AL, Zhao $Y$. The prevalence of hepatitis B core antibody in vaccinated Chinese children: a hospital-based study. Vaccine. 2019;37(3): 458-63. https://doi.org/10.1016/j.vaccine.2018.11.067.

34. Gore C, Wiktor S, Goldberg D, Hutchinson S, Onate Moreno JA, Peck R. Roundtable discussion: how the world hepatitis summit 2015 strengthened stakeholders' efforts to combat viral hepatitis. Hepatol Med Policy. 2016;1(1): 12. https://doi.org/10.1186/s41124-016-0018-4

\section{Publisher's Note}

Springer Nature remains neutral with regard to jurisdictional claims in published maps and institutional affiliations.

Ready to submit your research? Choose BMC and benefit from:

- fast, convenient online submission

- thorough peer review by experienced researchers in your field

- rapid publication on acceptance

- support for research data, including large and complex data types

- gold Open Access which fosters wider collaboration and increased citations

- maximum visibility for your research: over $100 \mathrm{M}$ website views per year

At $\mathrm{BMC}$, research is always in progress.

Learn more biomedcentral.com/submissions 